\title{
Mechanism and Chemoselectivity of the Pd(II)-catalyzed Allylation of Aldehydes: a Density Functional Theory Study
}

\author{
Fabio Pichierri ${ }^{\mathrm{a},{ }^{*}}$ and Yoshinori Yamamoto ${ }^{\mathrm{b}}$ \\ ${ }^{a}$ COE Laboratory, Tohoku University, IMRAM, 2-1-1 Katahira, Sendai 980-8577, Japan \\ ${ }^{b}$ Department of Chemistry, Graduate School of Science, Tohoku University, Sendai 980-8578, Japan \\ fabio@tagen.tohoku.ac.jp
}

Supporting Information Available

\section{Contents:}

Table S1: electronic energy (E), zero-point energy correction (ZPE),

ZPE-corrected electronic energy, and Gibbs free energy $(G)$ of the

DFT-optimized geometries labeled in the text...................................

Table S2: Cartesian coordinates of the DFT-optimized geometries

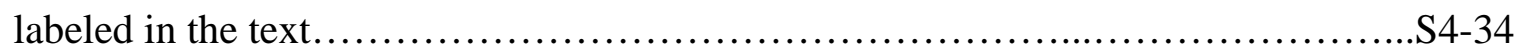


Table S1 containing the electronic energy (E), zero-point energy correction (ZPE), ZPE-corrected electronic energy, and Gibbs free energy (G) of the structures labeled in the text. All the energies are given in hartrees.

$\begin{array}{llccc}\text { molecule } & \mathrm{E} & \mathrm{ZPE} & \mathrm{E}+\mathrm{ZPE} & \mathrm{G} \\ ==================================================== \\ \mathrm{CH}_{2} \mathrm{O} & -114.385178 & 0.026988 & -114.358190 & -114.379201 \\ \mathrm{PH}_{3} & -342.954303 & 0.024451 & -342.929852 & -342.949851\end{array}$

Pd complexes

\begin{tabular}{|c|c|c|c|c|}
\hline 1 & -5173.650999 & 0.141447 & -5173.509553 & -5173.542835 \\
\hline 1-r & -5288.020966 & 0.170661 & -5287.850286 & -5287.889247 \\
\hline 1-ts & -5288.019748 & 0.171464 & -5287.848284 & -5287.885402 \\
\hline 1-p & -5288.063905 & 0.175832 & -5287.888073 & -5287.924239 \\
\hline 1: $\mathrm{CH}_{2} \mathrm{O}$ & -5288.039672 & 0.169657 & -5287.870016 & -5287.911480 \\
\hline \multicolumn{5}{|c|}{ with the SDD basis set for Pd only: } \\
\hline 1 & -362.223869 & 0.141766 & -362.082103 & -362.115157 \\
\hline 1-r & -476.592835 & 0.171249 & -476.421585 & -476.459918 \\
\hline 1-ts & -476.592133 & 0.171832 & -476.420300 & -476.457087 \\
\hline 1-p & -476.638676 & 0.176241 & -476.462434 & -476.498247 \\
\hline 2 & -5212.919863 & 0.169662 & -5212.750201 & -5212.785566 \\
\hline 2a-r & -5327.291786 & 0.198907 & -5327.092879 & -5327.134028 \\
\hline $2 a-t s$ & -5327.291172 & 0.199475 & -5327.091697 & -5327.131147 \\
\hline 2a-p & -5327.338822 & 0.204072 & -5327.134750 & -5327.172607 \\
\hline $2 b-\mathbf{r}$ & -5327.291008 & 0.198924 & -5327.092083 & -5327.132575 \\
\hline $2 b-t s$ & -5327.290024 & 0.199635 & -5327.090388 & -5327.129305 \\
\hline $2 \mathbf{b}-\mathbf{p}$ & -5327.334114 & 0.204042 & -5327.130072 & -5327.168236 \\
\hline $2 c-r$ & -5327.290282 & 0.198890 & -5327.091391 & -5327.132707 \\
\hline $2 c-t s$ & -5327.288112 & 0.199844 & -5327.088269 & -5327.127387 \\
\hline 2c-p & -5327.331671 & 0.204058 & -5327.127613 & -5327.165562 \\
\hline $2 d-r$ & -5327.283580 & 0.198360 & -5327.085220 & -5327.127007 \\
\hline
\end{tabular}




$\begin{array}{lllll}\text { 2d-ts } & -5327.281885 & 0.199771 & -5327.082114 & -5327.120860 \\ \text { 2d-p } & -5327.331843 & 0.204184 & -5327.127659 & -5327.165735 \\ & & & & \\ \text { 3 } & -5212.922328 & 0.169468 & -5212.752860 & -5212.788078 \\ \text { 3a-r } & -5327.292588 & 0.198682 & -5327.093906 & -5327.134694 \\ \text { 3a-ts } & -5327.291625 & 0.199375 & -5327.092250 & -5327.131401 \\ \text { 3a-p } & -5327.336262 & 0.203772 & -5327.132489 & -5327.170570 \\ \text { 3b-r } & -5327.294061 & 0.199261 & -5327.094800 & -5327.135048 \\ \text { 3b-ts } & -5327.293873 & 0.199497 & -5327.094376 & -5327.133258 \\ \text { 3b-p } & -5327.335065 & 0.203840 & -5327.131225 & -5327.169081 \\ & & & & \\ \text { 4 } & -5288.066507 & 0.174662 & -5287.891844 & -5287.928539 \\ \text { 4a-r } & -5402.437482 & 0.204035 & -5402.233447 & -5402.275613 \\ \text { 4a-ts } & -5402.436253 & 0.204690 & -5402.231564 & -5402.272033 \\ \text { 4a-p } & -5402.481389 & 0.209037 & -5402.272352 & -5402.312032 \\ \text { 4b-p1 } & -5402.482355 & 0.209081 & -5402.273274 & -5402.312769 \\ \text { 4b-p2 } & -5402.478050 & 0.208824 & -5402.269227 & -5402.309101\end{array}$

Pt complexes \$DD basis set for Pt only)

\begin{tabular}{lllll}
\hline $\mathbf{5}$ & -353.703243 & 0.142548 & -353.560695 & -353.594204 \\
$\mathbf{5 - r}$ & -468.071414 & 0.172305 & -467.899109 & -467.937578 \\
$\mathbf{5 - t s}$ & -468.070866 & 0.172783 & -467.898083 & -467.935105 \\
$\mathbf{5 - p}$ & -468.121632 & 0.177170 & -467.944461 & -467.980354
\end{tabular}

Pd complexes with coordinated phosphine (Szabo's mechanism)

\begin{tabular}{lllll}
\hline sz-1 & -5516.608602 & 0.168522 & -5516.440081 & -5516.479345 \\
sz-2 & -5516.606468 & 0.168092 & -5516.438376 & -5516.479168 \\
sz-r & -5630.997866 & 0.196569 & -5630.801297 & -5630.848953 \\
sz-ts & -5630.989026 & 0.200152 & -5630.788874 & -5630.829961 \\
sz-p & -5631.025557 & 0.201970 & -5630.823587 & -5630.866643 \\
========================================================
\end{tabular}


Table S2 containing the DFT-optimized geometries (Cartesian coordinates) of the structures labeled in the text (the coordinates of the Pd complexes with the SDD basis-set on Pd only are not reported in this table). “XX” represents a dummy atom.

$\begin{array}{cccc}\mathrm{CH}_{2} \mathrm{O} & & & \\ \mathrm{C} & 0.000000 & 0.000000 & 0.001288 \\ \mathrm{O} & 0.000000 & 0.000000 & 1.208186 \\ \mathrm{H} & 0.941828 & 0.000000 & -0.583223 \\ \mathrm{H} & -0.941828 & 0.000000 & -0.583223\end{array}$

$\mathrm{PH}_{3}$

$\begin{array}{lrrr}\mathrm{P} & -0.109442 & 0.189559 & -0.077387 \\ \mathrm{H} & 0.055361 & -0.095888 & 1.304765 \\ \mathrm{H} & 1.248598 & -0.095888 & -0.382727 \\ \mathrm{H} & -0.541257 & -1.129262 & -0.382727\end{array}$

\section{1}

Pd $\quad 0.000000 \quad 0.000000 \quad 0.000000$

C $\quad 1.617957 \quad-1.146811 \quad 0.992092$

C $\quad 0.914647 \quad-1.970587 \quad 0.091167$

$\begin{array}{llll}\text { C } & -0.479022 & -2.157324 & 0.181185\end{array}$

$\mathrm{H} \quad 2.664142 \quad-0.922715 \quad 0.805748$

$\mathrm{H} \quad 1.295083 \quad-1.067762 \quad 2.029246$

$\mathrm{H} \quad-0.999051 \quad-2.686790 \quad-0.611544$

$\mathrm{H} \quad-0.970167 \quad-2.160963 \quad 1.153331$

C $\quad-1.617957 \quad 1.146811 \quad-0.992092$

$\begin{array}{llll}\text { C } & -0.914647 & 1.970587 & -0.091167\end{array}$

$\begin{array}{llll}\text { C } & 0.479022 & 2.157324 & -0.181185\end{array}$

$\mathrm{H} \quad-2.664142 \quad 0.922715 \quad-0.805748$

$\mathrm{H} \quad-1.295083 \quad 1.067762 \quad-2.029246$

$\begin{array}{llll}\mathrm{H} & 0.999051 & 2.686790 & 0.611544\end{array}$

$\mathrm{H} \quad 0.970167 \quad 2.160963 \quad-1.153331$

H $\quad 1.407897 \quad-2.246069 \quad-0.841149$ 


$\begin{array}{cccc}\text { H } & -1.407897 & 2.246069 & 0.841149 \\ \text { XX } & 0.974848 & -0.219636 & -0.037825 \\ & & & \\ \text { 1-r } & & & \\ \text { Pd } & -0.457802 & 0.023223 & -0.074065 \\ \text { C } & -2.535251 & 1.049159 & -0.192490 \\ \text { C } & -2.538512 & -0.113651 & 0.568138 \\ \text { C } & -2.053548 & -1.341768 & 0.022176 \\ \text { H } & -2.735097 & 2.009834 & 0.273308 \\ \text { H } & -2.610821 & 1.010151 & -1.277628 \\ \text { H } & -1.960004 & -2.203078 & 0.678754 \\ \text { H } & -2.268339 & -1.580206 & -1.019776 \\ \text { H } & -2.665194 & -0.032425 & 1.646975 \\ \text { C } & 1.014595 & -1.552855 & 0.014471 \\ \text { H } & 1.016428 & -1.884812 & 1.057877 \\ \text { H } & 0.611795 & -2.334205 & -0.635283 \\ \text { C } & 2.302319 & -1.058936 & -0.441014 \\ \text { C } & 3.330005 & -0.605822 & 0.319754 \\ \text { H } & 2.424424 & -0.982373 & -1.523772 \\ \text { H } & 4.257048 & -0.263031 & -0.132886 \\ \text { H } & 3.312417 & -0.691752 & 1.405425 \\ \text { O } & 1.001814 & 1.616484 & -0.420727 \\ \text { C } & 1.956409 & 1.797503 & 0.329325 \\ \text { H } & 2.765231 & 2.481933 & 0.031197 \\ \text { H } & 1.997510 & 1.370549 & 1.339137\end{array}$

1-ts

$\begin{array}{rrrc}\text { Pd } & -0.447482 & 0.011319 & -0.060407 \\ \text { C } & -2.443871 & 1.058142 & -0.158461 \\ \text { C } & -2.515774 & -0.130544 & 0.569486 \\ \text { C } & -2.069281 & -1.345208 & -0.021143 \\ \text { H } & -2.592248 & 2.012841 & 0.337763 \\ \text { H } & -2.547304 & 1.056460 & -1.242130\end{array}$




$\begin{array}{lrrr}\mathrm{H} & -1.992916 & -2.234073 & 0.600004 \\ \mathrm{H} & -2.268010 & -1.533262 & -1.076282 \\ \mathrm{H} & -2.645187 & -0.081665 & 1.649573 \\ \mathrm{C} & 1.049316 & -1.617107 & 0.049883 \\ \mathrm{H} & 1.042901 & -1.893161 & 1.107670 \\ \mathrm{H} & 0.616174 & -2.386401 & -0.590557 \\ \mathrm{C} & 2.253475 & -1.035467 & -0.439027 \\ \mathrm{C} & 3.212322 & -0.353205 & 0.278524 \\ \mathrm{H} & 2.348391 & -0.993996 & -1.526046 \\ \mathrm{H} & 4.113044 & -0.011887 & -0.225008 \\ \mathrm{H} & 3.282879 & -0.482173 & 1.356676 \\ \mathrm{O} & 1.022305 & 1.528071 & -0.392250 \\ \mathrm{C} & 2.005196 & 1.582885 & 0.384552 \\ \mathrm{H} & 2.836078 & 2.259561 & 0.140375 \\ \mathrm{H} & 1.909815 & 1.291022 & 1.437162\end{array}$

$\begin{array}{rrrc}\text { 1-p } & & & \\ \text { Pd } & -0.114356 & 0.209358 & -0.000830 \\ \text { C } & -2.132004 & 0.978954 & 0.144579 \\ \text { C } & -2.119810 & -0.375048 & 0.555954 \\ \text { C } & -1.560549 & -1.353024 & -0.302966 \\ \text { H } & -2.358749 & 1.724355 & 0.901659 \\ \text { H } & -2.254721 & 1.299392 & -0.887639 \\ \text { H } & -1.391244 & -2.373627 & 0.028156 \\ \text { H } & -1.700010 & -1.227800 & -1.377332 \\ \text { H } & -2.247131 & -0.615802 & 1.609757 \\ \text { C } & 1.548976 & -1.459624 & 0.204956 \\ \text { H } & 1.539367 & -1.732209 & 1.258238 \\ \text { H } & 1.296670 & -2.215311 & -0.533650 \\ \text { C } & 2.111909 & -0.290146 & -0.237629 \\ \text { C } & 2.712967 & 0.816840 & 0.569977 \\ \text { H } & 2.227775 & -0.183133 & -1.316714 \\ \text { H } & 3.809177 & 0.734275 & 0.587518\end{array}$




$\begin{array}{lllr}\mathrm{H} & 2.377640 & 0.696653 & 1.609356 \\ \mathrm{O} & 0.881735 & 1.963742 & -0.530580 \\ \mathrm{C} & 2.129339 & 2.170207 & 0.039396 \\ \mathrm{H} & 2.803013 & 2.573789 & -0.745626 \\ \mathrm{H} & 2.104681 & 2.937891 & 0.833932\end{array}$

\begin{tabular}{cccc}
\multicolumn{1}{c}{$: \mathrm{CH}_{2} \mathrm{O}$} & & \\
$\mathrm{C}$ & 1.912260 & 0.431358 & 0.375533 \\
$\mathrm{C}$ & 1.386692 & 1.521399 & -0.346380 \\
$\mathrm{C}$ & 0.850075 & 1.362758 & -1.638931 \\
$\mathrm{H}$ & 2.202150 & 0.573684 & 1.411980 \\
$\mathrm{H}$ & 2.407640 & -0.384629 & -0.149549 \\
$\mathrm{H}$ & 0.326700 & 2.191718 & -2.106103 \\
$\mathrm{H}$ & 1.269737 & 0.623820 & -2.320384 \\
$\mathrm{C}$ & -2.259045 & -0.426593 & -0.886434 \\
$\mathrm{C}$ & -1.722359 & -1.519340 & -0.180988 \\
$\mathrm{C}$ & -1.238221 & -1.396022 & 1.138366 \\
$\mathrm{H}$ & -2.496821 & -0.534105 & -1.940302 \\
$\mathrm{H}$ & -2.797215 & 0.358676 & -0.357430 \\
$\mathrm{H}$ & -0.720521 & -2.233385 & 1.596861 \\
$\mathrm{H}$ & -1.728535 & -0.710672 & 1.828677 \\
$\mathrm{H}$ & 1.153432 & 2.431605 & 0.205819 \\
$\mathrm{H}$ & -1.432591 & -2.402264 & -0.751044 \\
$\mathrm{Pd}$ & -0.181832 & 0.012274 & -0.220099 \\
$\mathrm{O}$ & 0.644003 & 2.510051 & 2.923402 \\
$\mathrm{C}$ & -0.194238 & 1.639765 & 2.891917 \\
$\mathrm{H}$ & -1.156577 & 1.774597 & 2.358220 \\
$\mathrm{H}$ & -0.049038 & 0.666209 & 3.399814
\end{tabular}

$\begin{array}{rrrc}2 & & & \\ \text { Pd } & -0.010787 & -0.016784 & 0.007958 \\ \text { C } & 1.622331 & -1.169818 & 0.987517 \\ \text { C } & 0.941750 & -1.972423 & 0.051643\end{array}$




$\begin{array}{crrc}\mathrm{C} & -0.449699 & -2.189703 & 0.115905 \\ \mathrm{H} & 2.668079 & -0.927663 & 0.821868 \\ \mathrm{H} & 1.284175 & -1.127042 & 2.022074 \\ \mathrm{H} & -0.946930 & -2.705803 & -0.699911 \\ \mathrm{H} & -0.952718 & -2.235508 & 1.080838 \\ \mathrm{C} & -1.639012 & 1.124462 & -0.930772 \\ \mathrm{C} & -0.941017 & 1.950618 & -0.022224 \\ \mathrm{C} & 0.445779 & 2.171434 & -0.122411 \\ \mathrm{H} & -2.678640 & 0.879852 & -0.734048 \\ \mathrm{H} & -1.338264 & 1.087987 & -1.977117 \\ \mathrm{H} & 0.885386 & 2.145166 & -1.121917 \\ \mathrm{H} & 1.451687 & -2.209217 & -0.882571 \\ \mathrm{H} & -1.428916 & 2.208500 & 0.919382 \\ \mathrm{C} & 1.212934 & 2.955635 & 0.905335 \\ \mathrm{H} & 2.247525 & 2.609328 & 0.976774 \\ \mathrm{H} & 1.243889 & 4.022458 & 0.645569 \\ \mathrm{H} & 0.761867 & 2.866239 & 1.897861\end{array}$

$\begin{array}{cccc}\text { 2a-r } & & & \\ \text { Pd } & -0.454723 & 0.006732 & -0.030233 \\ \text { C } & -2.544852 & 1.057352 & -0.130283 \\ \text { C } & -2.544072 & -0.120218 & 0.607103 \\ \text { C } & -2.053839 & -1.339574 & 0.039613 \\ \text { H } & -2.587381 & 0.982054 & -1.218419 \\ \text { H } & -1.968026 & -2.211584 & 0.683471 \\ \text { H } & -2.277136 & -1.565170 & -1.003666 \\ \text { H } & -2.676467 & -0.057110 & 1.687569 \\ \text { C } & 1.036345 & -1.564379 & -0.000400 \\ \text { H } & 1.056581 & -1.917500 & 1.035767 \\ \text { H } & 0.625273 & -2.331094 & -0.662035 \\ \text { C } & 2.305430 & -1.042213 & -0.466011 \\ \text { C } & 3.338304 & -0.577640 & 0.284974 \\ \text { H } & 2.407247 & -0.940796 & -1.548905\end{array}$




$\begin{array}{lccc}\mathrm{H} & 4.251505 & -0.214924 & -0.179977 \\ \mathrm{H} & 3.347849 & -0.693838 & 1.367786 \\ \mathrm{O} & 0.999441 & 1.618847 & -0.329111 \\ \mathrm{C} & 1.973165 & 1.749666 & 0.410679 \\ \mathrm{H} & 2.795766 & 2.421106 & 0.121559 \\ \mathrm{H} & 2.007794 & 1.307906 & 1.414238 \\ \mathrm{C} & -2.840751 & 2.404925 & 0.454615 \\ \mathrm{H} & -2.167879 & 3.166094 & 0.048287 \\ \mathrm{H} & -3.864394 & 2.720609 & 0.215303 \\ \mathrm{H} & -2.733754 & 2.404062 & 1.542868\end{array}$

\section{2a-ts}

$\begin{array}{cccc}\text { Pd } & -0.269028 & -0.185193 & -0.091062 \\ \mathrm{C} & -2.291185 & 0.875413 & -0.189355 \\ \mathrm{C} & -2.345963 & -0.314393 & 0.536909 \\ \mathrm{C} & -1.885296 & -1.529722 & -0.049968 \\ \mathrm{H} & -2.357365 & 0.813948 & -1.277270 \\ \mathrm{H} & -1.811311 & -2.415260 & 0.576567 \\ \mathrm{H} & -2.094004 & -1.727962 & -1.101593 \\ \mathrm{H} & -2.482014 & -0.262870 & 1.617201 \\ \mathrm{C} & 1.252984 & -1.787117 & -0.030388 \\ \mathrm{H} & 1.267494 & -2.088843 & 1.020421 \\ \mathrm{H} & 0.823976 & -2.551158 & -0.680251 \\ \mathrm{C} & 2.448481 & -1.185076 & -0.522461 \\ \mathrm{C} & 3.417947 & -0.523358 & 0.196253 \\ \mathrm{H} & 2.528664 & -1.118417 & -1.609561 \\ \mathrm{H} & 4.306219 & -0.156078 & -0.311030 \\ \mathrm{H} & 3.499974 & -0.667399 & 1.271769 \\ \mathrm{O} & 1.176692 & 1.379979 & -0.391619 \\ \mathrm{C} & 2.169471 & 1.431471 & 0.367762 \\ \mathrm{H} & 2.996502 & 2.112101 & 0.120975 \\ \mathrm{H} & 2.103406 & 1.099138 & 1.410720 \\ \mathrm{C} & -2.532823 & 2.226410 & 0.411800\end{array}$




$\begin{array}{llll}\mathrm{H} & -1.833767 & 2.963284 & 0.006137 \\ \mathrm{H} & -3.546195 & 2.579584 & 0.181402 \\ \mathrm{H} & -2.417705 & 2.211153 & 1.498955\end{array}$

$\begin{array}{crrr}\text { 2a-p } & & & \\ \text { Pd } & 0.356825 & 0.041807 & 0.077489 \\ \text { C } & 2.012220 & -1.388209 & 0.144570 \\ \text { C } & 2.492899 & -0.115987 & 0.507573 \\ \text { C } & 2.287141 & 0.972592 & -0.365301 \\ \text { H } & 1.996331 & -1.635172 & -0.919004 \\ \text { H } & 2.514837 & 1.977971 & -0.020329 \\ \text { H } & 2.338828 & 0.823013 & -1.443486 \\ \text { H } & 2.742955 & 0.073537 & 1.551016 \\ \text { C } & -0.759669 & 2.055768 & 0.125893 \\ \text { H } & -0.775202 & 2.324217 & 1.180476 \\ \text { H } & -0.215916 & 2.720471 & -0.539395 \\ \text { C } & -1.609499 & 1.101873 & -0.367940 \\ \text { C } & -2.589982 & 0.295807 & 0.437848 \\ \text { H } & -1.680874 & 0.991714 & -1.452714 \\ \text { H } & -3.613293 & 0.548575 & 0.124465 \\ \text { H } & -2.487517 & 0.538193 & 1.501154 \\ \text { O } & -0.970309 & -1.461059 & 0.551355 \\ \text { C } & -2.293974 & -1.188925 & 0.223904 \\ \text { H } & -2.528658 & -1.438306 & -0.830379 \\ \text { H } & -2.971592 & -1.798859 & 0.846044 \\ \text { C } & 1.866052 & -2.533359 & 1.093257 \\ \text { H } & 0.825272 & -2.881170 & 1.069767 \\ \text { H } & 2.516684 & -3.367425 & 0.806560 \\ \text { H } & 2.103967 & -2.239429 & 2.119220\end{array}$

\section{2b-r}

$\begin{array}{llll}\text { Pd } & -0.439837 & 0.027915 & -0.055782\end{array}$

$\begin{array}{llll}\text { C } & -2.478968 & 1.062635 & -0.120670\end{array}$ 


$\begin{array}{lrrr}\mathrm{C} & -2.497073 & -0.107379 & 0.633856 \\ \mathrm{C} & -2.059364 & -1.346683 & 0.075440 \\ \mathrm{H} & -2.599832 & 1.034591 & -1.202181 \\ \mathrm{H} & -2.276654 & -1.505433 & -0.983750 \\ \mathrm{H} & -2.604732 & -0.034350 & 1.716513 \\ \mathrm{C} & 1.046816 & -1.547914 & 0.000989 \\ \mathrm{H} & 1.023238 & -1.903836 & 1.036672 \\ \mathrm{H} & 0.646563 & -2.305287 & -0.678712 \\ \mathrm{C} & 2.343120 & -1.053064 & -0.420062 \\ \mathrm{C} & 3.349401 & -0.588249 & 0.363697 \\ \mathrm{H} & 2.491155 & -0.974240 & -1.499543 \\ \mathrm{H} & 4.286346 & -0.245973 & -0.068436 \\ \mathrm{H} & 3.306104 & -0.669452 & 1.448989 \\ \mathrm{O} & 1.027739 & 1.601861 & -0.459568 \\ \mathrm{C} & 1.993166 & 1.797065 & 0.274602 \\ \mathrm{H} & 2.815503 & 2.446084 & -0.062916 \\ \mathrm{H} & 2.028554 & 1.429878 & 1.307995 \\ \mathrm{H} & -2.647112 & 2.023022 & 0.358275 \\ \mathrm{C} & -1.995744 & -2.598805 & 0.903635 \\ \mathrm{H} & -2.954804 & -3.132662 & 0.875379 \\ \mathrm{H} & -1.231282 & -3.284932 & 0.528362 \\ \mathrm{H} & -1.762326 & -2.378996 & 1.949420\end{array}$

$\begin{array}{cccc}\text { 2b-ts } & & & \\ \text { Pd } & -0.306666 & 0.197774 & -0.122942 \\ \text { C } & -2.274006 & 1.252706 & -0.180908 \\ \text { C } & -2.353091 & 0.074066 & 0.566483 \\ \text { C } & -1.957052 & -1.163695 & -0.011253 \\ \text { H } & -2.422752 & 1.241096 & -1.259417 \\ \text { H } & -2.164549 & -1.297242 & -1.075644 \\ \text { H } & -2.458403 & 0.137916 & 1.649613 \\ \text { C } & 1.206698 & -1.424298 & -0.033710 \\ \text { H } & 1.180410 & -1.711336 & 1.021214\end{array}$




$\begin{array}{lrrr}\mathrm{H} & 0.778145 & -2.181714 & -0.691468 \\ \mathrm{C} & 2.421589 & -0.848201 & -0.500278 \\ \mathrm{C} & 3.362884 & -0.155195 & 0.228942 \\ \mathrm{H} & 2.540058 & -0.817159 & -1.585439 \\ \mathrm{H} & 4.276835 & 0.177798 & -0.255933 \\ \mathrm{H} & 3.401204 & -0.258234 & 1.311695 \\ \mathrm{O} & 1.161513 & 1.703268 & -0.531098 \\ \mathrm{C} & 2.152285 & 1.799797 & 0.230173 \\ \mathrm{H} & 2.985591 & 2.453025 & -0.064374 \\ \mathrm{H} & 2.070130 & 1.567947 & 1.298852 \\ \mathrm{H} & -2.392521 & 2.215485 & 0.307977 \\ \mathrm{C} & -1.916359 & -2.429745 & 0.796144 \\ \mathrm{H} & -2.894442 & -2.928170 & 0.780726 \\ \mathrm{H} & -1.186631 & -3.136687 & 0.392608 \\ \mathrm{H} & -1.652949 & -2.234379 & 1.839389\end{array}$

$\begin{array}{crrc}\text { 2b-p } & & & \\ \text { Pd } & 0.317776 & 0.003415 & 0.154662 \\ \text { C } & 1.969445 & -1.361406 & 0.135872 \\ \text { C } & 2.479285 & -0.094986 & 0.489539 \\ \text { C } & 2.268364 & 0.994272 & -0.371765 \\ \text { H } & 1.957855 & -1.675354 & -0.906862 \\ \text { H } & 2.217223 & 0.779718 & -1.441450 \\ \text { H } & 2.772107 & 0.095545 & 1.521836 \\ \text { C } & -0.808275 & 2.005740 & 0.394737 \\ \text { H } & -0.882431 & 2.137873 & 1.472481 \\ \text { H } & -0.217050 & 2.736896 & -0.148584 \\ \text { C } & -1.645160 & 1.146713 & -0.262985 \\ \text { C } & -2.673608 & 0.266787 & 0.384025 \\ \text { H } & -1.651105 & 1.169750 & -1.355301 \\ \text { H } & -3.667316 & 0.516180 & -0.014107 \\ \text { H } & -2.683342 & 0.435091 & 1.466078 \\ \text { O } & -1.043885 & -1.477078 & 0.576134\end{array}$




$\begin{array}{cccc}\mathrm{C} & -2.317498 & -1.197242 & 0.105951 \\ \mathrm{H} & -2.424446 & -1.374983 & -0.983116 \\ \mathrm{H} & -3.051216 & -1.857270 & 0.601236 \\ \mathrm{H} & 1.928336 & -2.158856 & 0.871430 \\ \mathrm{C} & 2.658010 & 2.396875 & -0.006315 \\ \mathrm{H} & 3.699999 & 2.591628 & -0.291091 \\ \mathrm{H} & 2.044851 & 3.135174 & -0.530998 \\ \mathrm{H} & 2.562172 & 2.577215 & 1.067826\end{array}$

$\begin{array}{crrr}\text { 2c-r } & & & \\ \text { Pd } & -0.476989 & 0.030373 & -0.082066 \\ \text { C } & -2.586865 & 1.015097 & -0.202096 \\ \text { C } & -2.547510 & -0.119604 & 0.597406 \\ \text { C } & -2.045499 & -1.357712 & 0.087972 \\ \text { H } & -2.673117 & 0.936059 & -1.284264 \\ \text { H } & -1.925857 & -2.192511 & 0.774003 \\ \text { H } & -2.274667 & -1.638849 & -0.940210 \\ \text { H } & -2.658667 & -0.002997 & 1.674791 \\ \text { C } & 1.028776 & -1.502443 & 0.030067 \\ \text { H } & 1.038611 & -1.822077 & 1.077692 \\ \text { H } & 0.640729 & -2.307413 & -0.600588 \\ \text { C } & 2.326706 & -1.026330 & -0.434526 \\ \text { C } & 3.364344 & -0.592195 & 0.321857 \\ \text { H } & 2.453303 & -0.966093 & -1.519183 \\ \text { H } & 3.279587 & -0.667920 & 1.408437 \\ \text { O } & 0.972488 & 1.620028 & -0.508024 \\ \text { C } & 1.945700 & 1.804674 & 0.214617 \\ \text { H } & 2.773929 & 2.442442 & -0.132794 \\ \text { H } & 1.992054 & 1.425325 & 1.243666 \\ \text { H } & -2.803292 & 1.986405 & 0.233155 \\ \text { C } & 4.692741 & -0.149401 & -0.215792 \\ \text { H } & 5.487903 & -0.855701 & 0.055732 \\ \text { H } & 4.675515 & -0.072796 & -1.307316\end{array}$


$\begin{array}{llll}\mathrm{H} & 5.000125 & 0.825939 & 0.184711\end{array}$

$\begin{array}{crrr}\text { 2c-ts } & & & \\ \text { Pd } & -0.766359 & -0.004620 & -0.030408 \\ \text { C } & -2.749411 & 1.048170 & -0.185599 \\ \text { C } & -2.835316 & -0.105679 & 0.596580 \\ \text { C } & -2.399180 & -1.349872 & 0.064331 \\ \text { H } & -2.853982 & 0.998006 & -1.267991 \\ \text { H } & -2.332396 & -2.209884 & 0.725962 \\ \text { H } & -2.593916 & -1.584002 & -0.982264 \\ \text { H } & -2.967433 & -0.005876 & 1.672839 \\ \text { C } & 0.743549 & -1.619985 & 0.164828 \\ \text { H } & 0.721595 & -1.844916 & 1.234290 \\ \text { H } & 0.308722 & -2.413490 & -0.444025 \\ \text { C } & 1.958060 & -1.079080 & -0.338826 \\ \text { C } & 2.916803 & -0.360027 & 0.350876 \\ \text { H } & 2.071670 & -1.102988 & -1.425983 \\ \text { H } & 2.913002 & -0.447242 & 1.438323 \\ \text { O } & 0.725092 & 1.456856 & -0.440629 \\ \text { C } & 1.717923 & 1.512287 & 0.332375 \\ \text { H } & 2.562902 & 2.157960 & 0.049215 \\ \text { H } & 1.599134 & 1.319063 & 1.406244 \\ \text { H } & -2.889474 & 2.025830 & 0.266355 \\ \text { C } & 4.231005 & 0.033220 & -0.268218 \\ \text { H } & 5.000980 & -0.731238 & -0.103114 \\ \text { H } & 4.133166 & 0.178391 & -1.348971 \\ \text { H } & 4.619442 & 0.966305 & 0.157479\end{array}$

$\begin{array}{cccc}\text { 2c-p } & & & \\ \text { C } & 2.000610 & -1.415654 & -0.001269 \\ \text { C } & 2.528339 & -0.161292 & 0.355269 \\ \text { C } & 2.263123 & 0.945692 & -0.471665 \\ \text { Pd } & 0.362311 & -0.004821 & 0.116810\end{array}$




$\begin{array}{lrrr}\mathrm{O} & -1.009468 & -1.441152 & 0.625899 \\ \mathrm{C} & -2.312795 & -1.116211 & 0.281182 \\ \mathrm{C} & -2.600354 & 0.364956 & 0.555928 \\ \mathrm{C} & -4.017537 & 0.781760 & 0.161430 \\ \mathrm{C} & -0.675751 & 2.038303 & 0.360711 \\ \mathrm{C} & -1.575967 & 1.171161 & -0.196692 \\ \mathrm{H} & 1.920583 & -1.700239 & -1.049187 \\ \mathrm{H} & 2.518445 & 1.943567 & -0.124656 \\ \mathrm{H} & 2.208859 & 0.819033 & -1.552412 \\ \mathrm{H} & 2.861067 & 0.005658 & 1.378227 \\ \mathrm{H} & -0.665874 & 2.215439 & 1.434589 \\ \mathrm{H} & -0.108219 & 2.730728 & -0.254547 \\ \mathrm{H} & -1.669494 & 1.155168 & -1.286257 \\ \mathrm{H} & -2.452217 & 0.533948 & 1.629839 \\ \mathrm{H} & -2.537061 & -1.309112 & -0.788746 \\ \mathrm{H} & -3.016025 & -1.740215 & 0.861074 \\ \mathrm{H} & 1.983096 & -2.228660 & 0.717831 \\ \mathrm{H} & -4.756863 & 0.193967 & 0.714793 \\ \mathrm{H} & -4.202392 & 1.839868 & 0.372420 \\ \mathrm{H} & -4.195610 & 0.616400 & -0.907159\end{array}$

\section{2d-r}

$\begin{array}{rrrc}\text { Pd } & -0.410991 & -0.052095 & -0.143293 \\ \text { C } & -2.461495 & 1.109153 & -0.045101 \\ \text { C } & -2.403141 & -0.006442 & 0.777493 \\ \text { C } & -2.062324 & -1.295102 & 0.258464 \\ \text { H } & -2.672216 & 1.012679 & -1.108580 \\ \text { H } & -1.939179 & -2.119553 & 0.956467 \\ \text { H } & -2.433306 & -1.580399 & -0.726528 \\ \text { H } & -2.377539 & 0.144437 & 1.856361 \\ \text { C } & 0.956547 & -1.705693 & -0.135152 \\ \text { H } & 0.839865 & -2.141318 & 0.863724 \\ \text { C } & 2.283549 & -1.111992 & -0.294029\end{array}$




$\begin{array}{lrrr}\mathrm{C} & 3.155463 & -0.765515 & 0.677836 \\ \mathrm{H} & 2.584512 & -0.909642 & -1.326142 \\ \mathrm{H} & 2.940688 & -0.951539 & 1.729523 \\ \mathrm{O} & 0.993829 & 1.582050 & -0.698613 \\ \mathrm{C} & 1.856865 & 2.019195 & 0.045312 \\ \mathrm{H} & 2.488441 & 2.860089 & -0.284895 \\ \mathrm{H} & 2.030440 & 1.604534 & 1.048234 \\ \mathrm{H} & -2.558747 & 2.102868 & 0.382611 \\ \mathrm{H} & 4.127202 & -0.338971 & 0.439538 \\ \mathrm{C} & 0.614638 & -2.719278 & -1.222045 \\ \mathrm{H} & 1.328997 & -3.556428 & -1.228163 \\ \mathrm{H} & -0.383676 & -3.143430 & -1.083795 \\ \mathrm{H} & 0.640156 & -2.267136 & -2.220214\end{array}$

\section{2d-ts}

$\begin{array}{llll}\text { Pd } & -0.492809 & 0.226156 & 0.001248\end{array}$

$\begin{array}{llll}\text { C } & -2.456846 & 1.339045 & 0.042442\end{array}$

$\begin{array}{llll}\text { C } & -2.505429 & 0.176104 & 0.812405\end{array}$

C $\quad-2.152895 \quad-1.070657 \quad 0.227361$

$\mathrm{H} \quad-2.653659 \quad 1.308979 \quad-1.027801$

$\mathrm{H} \quad-2.054897 \quad-1.941069 \quad 0.871209$

$\mathrm{H} \quad-2.444740 \quad-1.283408 \quad-0.801200$

$\mathrm{H} \quad-2.539035 \quad 0.262294 \quad 1.897454$

$\begin{array}{llll}\text { C } & 0.982138 & -1.455049 & 0.003056\end{array}$

$\mathrm{H} \quad 0.926149 \quad-1.759203 \quad 1.054075$

C $\quad 2.204305 \quad-0.794581 \quad-0.331373$

$\begin{array}{llll}\text { C } & 3.076798 & -0.126777 & 0.496534\end{array}$

$\mathrm{H} \quad 2.402752 \quad-0.707250 \quad-1.403277$

$\mathrm{H} \quad 3.034595 \quad-0.272276 \quad 1.574367$

$\begin{array}{llll}\text { O } & 0.960254 & 1.745785 & -0.399207\end{array}$

$\begin{array}{llll}\mathrm{C} & 1.897900 & 1.862248 & 0.421997\end{array}$

$\begin{array}{llll}\mathrm{H} & 2.738491 & 2.527585 & 0.178209\end{array}$

$\mathrm{H} \quad 1.763283 \quad 1.618040 \quad 1.482122$ 


$\begin{array}{lrrr}\mathrm{H} & -2.525857 & 2.313028 & 0.518094 \\ \mathrm{H} & 4.014515 & 0.248218 & 0.095055 \\ \mathrm{C} & 0.548514 & -2.553455 & -0.959136 \\ \mathrm{H} & 1.251443 & -3.398451 & -0.941760 \\ \mathrm{H} & -0.438866 & -2.952210 & -0.714313 \\ \mathrm{H} & 0.505660 & -2.188594 & -1.991189\end{array}$

$\begin{array}{crrr}\text { 2d-p } & & & \\ \text { Pd } & 0.388604 & -0.028456 & 0.094594 \\ \text { C } & 2.006495 & -1.447023 & 0.219397 \\ \text { C } & 2.530426 & -0.171265 & 0.499862 \\ \text { C } & 2.343514 & 0.856082 & -0.441079 \\ \text { H } & 2.001721 & -1.826619 & -0.801116 \\ \text { H } & 2.359441 & 0.629887 & -1.506817 \\ \text { H } & 2.794477 & 0.084472 & 1.524523 \\ \text { C } & -0.745970 & 2.057187 & 0.057149 \\ \text { H } & -0.821886 & 2.267045 & 1.125382 \\ \mathrm{C} & -1.555135 & 1.077124 & -0.452728 \\ \mathrm{C} & -2.568953 & 0.286377 & 0.326785 \\ \text { H } & -1.594617 & 0.965858 & -1.540328 \\ \text { H } & -3.572981 & 0.521891 & -0.055609 \\ \text { H } & -2.532772 & 0.571178 & 1.383940 \\ \mathrm{O} & -0.972570 & -1.458876 & 0.649582 \\ \mathrm{C} & -2.259630 & -1.203127 & 0.197510 \\ \mathrm{H} & -2.410120 & -1.498528 & -0.860487 \\ \mathrm{H} & -2.984536 & -1.789330 & 0.788920 \\ \mathrm{H} & 1.915079 & -2.189552 & 1.005883 \\ \mathrm{H} & 2.596736 & 1.877187 & -0.170519 \\ \mathrm{C} & -0.066622 & 3.107314 & -0.776597 \\ \mathrm{H} & -0.710857 & 3.994437 & -0.830666 \\ \mathrm{H} & 0.883834 & 3.430153 & -0.345527 \\ \mathrm{H} & 0.114238 & 2.762467 & -1.797886\end{array}$




$\begin{array}{cccc}3 & & & \\ \mathrm{Pd} & 0.011666 & -0.022970 & -0.008907 \\ \mathrm{C} & 1.582624 & -1.231291 & 0.991039 \\ \mathrm{C} & 0.880079 & -2.015244 & 0.054551 \\ \mathrm{C} & -0.518735 & -2.174965 & 0.109135 \\ \mathrm{H} & 2.637300 & -1.027134 & 0.832420 \\ \mathrm{H} & 1.238786 & -1.171666 & 2.022742 \\ \mathrm{H} & -1.031411 & -2.674691 & -0.707341 \\ \mathrm{H} & -1.030860 & -2.191065 & 1.070208 \\ \mathrm{C} & -1.553743 & 1.189942 & -0.982090 \\ \mathrm{C} & -0.886758 & 1.975671 & -0.014525 \\ \mathrm{C} & 0.515861 & 2.122883 & -0.112310 \\ \mathrm{H} & -2.610281 & 0.968400 & -0.855937 \\ \mathrm{H} & -1.185371 & 1.160215 & -2.006669 \\ \mathrm{H} & 1.058574 & 2.622237 & 0.686032 \\ \mathrm{H} & 0.993836 & 2.142743 & -1.090828 \\ \mathrm{H} & 1.387425 & -2.277028 & -0.874246 \\ \mathrm{C} & -1.609958 & 2.419549 & 1.230519 \\ \mathrm{H} & -2.456322 & 1.767061 & 1.460383 \\ \mathrm{H} & -0.943088 & 2.448978 & 2.096122 \\ \mathrm{H} & -2.001643 & 3.431703 & 1.077185\end{array}$

$\begin{array}{crrr}\text { 3a-r } & & & \\ \text { Pd } & -0.440314 & 0.036614 & -0.068472 \\ \text { C } & -2.499344 & 1.059106 & -0.159724 \\ \text { C } & -2.521448 & -0.092269 & 0.626044 \\ \text { C } & -2.045650 & -1.306150 & 0.028148 \\ \text { H } & -2.691301 & 2.031873 & 0.286013 \\ \text { H } & -2.584010 & 0.997879 & -1.243171 \\ \text { H } & -1.951011 & -2.191754 & 0.653254 \\ \text { H } & -2.281891 & -1.508239 & -1.016914 \\ \text { C } & 1.026908 & -1.550614 & 0.004583 \\ \text { H } & 1.030355 & -1.891988 & 1.044994\end{array}$




$\begin{array}{lrrr}\mathrm{H} & 0.614326 & -2.322405 & -0.650447 \\ \mathrm{C} & 2.315137 & -1.061712 & -0.450835 \\ \mathrm{C} & 3.347244 & -0.614710 & 0.309019 \\ \mathrm{H} & 2.434308 & -0.977367 & -1.533384 \\ \mathrm{H} & 4.274361 & -0.274762 & -0.145562 \\ \mathrm{H} & 3.334585 & -0.709377 & 1.394002 \\ \mathrm{O} & 1.035669 & 1.615895 & -0.402772 \\ \mathrm{C} & 1.996319 & 1.774016 & 0.345774 \\ \mathrm{H} & 2.818092 & 2.443364 & 0.049089 \\ \mathrm{H} & 2.026793 & 1.348884 & 1.356739 \\ \mathrm{C} & -2.789379 & -0.024690 & 2.105245 \\ \mathrm{H} & -3.832267 & -0.303193 & 2.296144 \\ \mathrm{H} & -2.154923 & -0.721394 & 2.659294 \\ \mathrm{H} & -2.634913 & 0.982880 & 2.499301\end{array}$

$\begin{array}{crrr}\text { 3a-ts } & & & \\ \text { Pd } & -0.255983 & 0.019154 & -0.234011 \\ \text { C } & -2.241931 & 1.061030 & -0.306329 \\ \text { C } & -2.319669 & -0.105521 & 0.464833 \\ \text { C } & -1.887139 & -1.313180 & -0.162752 \\ \text { H } & -2.379595 & 2.033981 & 0.158610 \\ \text { H } & -2.366518 & 1.022853 & -1.387111 \\ \text { H } & -1.803665 & -2.215926 & 0.438799 \\ \text { H } & -2.115908 & -1.479998 & -1.215435 \\ \text { C } & 1.231655 & -1.618045 & -0.117299 \\ \text { H } & 1.220667 & -1.896701 & 0.939838 \\ \text { H } & 0.794529 & -2.384008 & -0.759147 \\ \text { C } & 2.444631 & -1.049307 & -0.601093 \\ \text { C } & 3.408012 & -0.377735 & 0.119006 \\ \text { H } & 2.544962 & -1.010706 & -1.687776 \\ \mathrm{H} & 4.313916 & -0.044100 & -0.380297 \\ \mathrm{H} & 3.468713 & -0.498063 & 1.198859 \\ \mathrm{O} & 1.227328 & 1.526056 & -0.572137\end{array}$




$\begin{array}{rrrc}\mathrm{C} & 2.207903 & 1.583150 & 0.205008 \\ \mathrm{H} & 3.047097 & 2.247029 & -0.046118 \\ \mathrm{H} & 2.111941 & 1.296584 & 1.259128 \\ \mathrm{C} & -2.578776 & -0.052609 & 1.945540 \\ \mathrm{H} & -3.639075 & -0.257418 & 2.133530 \\ \mathrm{H} & -1.996008 & -0.805659 & 2.482007 \\ \mathrm{H} & -2.349880 & 0.932057 & 2.360302\end{array}$

$\begin{array}{cccc}\text { 3a-p } & & & \\ \text { Pd } & -0.307436 & -0.015540 & 0.002362 \\ \text { C } & -1.979788 & -1.366205 & 0.096039 \\ \text { C } & -2.455078 & -0.154133 & -0.453052 \\ \text { C } & -2.225638 & 1.001041 & 0.325676 \\ \text { H } & -1.915255 & -2.259259 & -0.519359 \\ \text { H } & -2.014984 & -1.530322 & 1.172027 \\ \text { H } & -2.425117 & 1.980540 & -0.103687 \\ \text { H } & -2.276131 & 0.943450 & 1.412549 \\ \text { C } & 0.808741 & 1.976029 & -0.340597 \\ \text { H } & 0.921833 & 2.036895 & -1.421354 \\ \text { H } & 0.194006 & 2.737376 & 0.130825 \\ \text { C } & 1.627219 & 1.167857 & 0.400871 \\ \text { C } & 2.685509 & 0.257912 & -0.150164 \\ \text { H } & 1.594571 & 1.262807 & 1.488815 \\ \text { H } & 3.662904 & 0.546971 & 0.261408 \\ \text { H } & 2.729301 & 0.351126 & -1.240500 \\ \text { O } & 1.075328 & -1.508408 & -0.268505 \\ \text { C } & 2.333253 & -1.186602 & 0.218171 \\ \text { H } & 2.409227 & -1.287603 & 1.319581 \\ \text { H } & 3.085464 & -1.873293 & -0.208175 \\ \text { C } & -2.891074 & -0.061884 & -1.888692 \\ \text { H } & -3.978124 & -0.191801 & -1.942062 \\ \text { H } & -2.651159 & 0.912229 & -2.322099 \\ \text { H } & -2.431327 & -0.843188 & -2.498310\end{array}$




$\begin{array}{crrc}\text { 3b-r } & & & \\ \text { Pd } & -0.236645 & 0.036170 & -0.274999 \\ \text { C } & -2.250057 & 1.099017 & -0.644987 \\ \text { C } & -2.380926 & -0.076270 & 0.086790 \\ \text { C } & -1.851552 & -1.303933 & -0.414773 \\ \text { H } & -2.487299 & 2.056725 & -0.190760 \\ \text { H } & -2.190170 & 1.078781 & -1.731668 \\ \text { H } & -1.857012 & -2.177332 & 0.232406 \\ \text { H } & -1.929942 & -1.520543 & -1.480320 \\ \text { C } & 1.206639 & -1.556157 & -0.018907 \\ \text { H } & 1.056578 & -1.908038 & 1.006324 \\ \text { H } & 0.897197 & -2.323006 & -0.734948 \\ \text { C } & 2.551930 & -1.059799 & -0.270725 \\ \text { C } & 3.389416 & -0.618040 & 0.710384 \\ \text { H } & 4.394364 & -0.272262 & 0.479642 \\ \text { H } & 3.147834 & -0.764042 & 1.761356 \\ \text { O } & 1.321486 & 1.554696 & -0.404083 \\ \text { C } & 2.154292 & 1.664533 & 0.500823 \\ \text { H } & 3.058223 & 2.269887 & 0.339494 \\ \text { H } & 1.957089 & 1.311569 & 1.520084 \\ \text { H } & -2.645738 & -0.009742 & 1.141301 \\ \text { C } & 2.957964 & -0.902902 & -1.709736 \\ \text { H } & 2.359289 & -0.111467 & -2.178391 \\ \text { H } & 4.014222 & -0.641816 & -1.814587 \\ \text { H } & 2.768413 & -1.824244 & -2.270736\end{array}$

\section{3b-ts}

$\begin{array}{rrrc}\text { Pd } & -0.627800 & 0.079756 & -0.014563 \\ \text { C } & -2.600697 & 1.152626 & -0.383594 \\ \text { C } & -2.765153 & -0.025352 & 0.342864 \\ \text { C } & -2.254670 & -1.254251 & -0.166887 \\ \text { H } & -2.813993 & 2.113192 & 0.076418 \\ \text { H } & -2.554515 & 1.136810 & -1.471072\end{array}$




$\begin{array}{cccc}\mathrm{H} & -2.268554 & -2.132659 & 0.473305 \\ \mathrm{H} & -2.325087 & -1.459891 & -1.235050 \\ \mathrm{C} & 0.827046 & -1.532692 & 0.258409 \\ \mathrm{H} & 0.674687 & -1.854468 & 1.292254 \\ \mathrm{H} & 0.506700 & -2.298363 & -0.451781 \\ \mathrm{C} & 2.133540 & -0.988414 & -0.014583 \\ \mathrm{C} & 2.937350 & -0.424451 & 0.947922 \\ \mathrm{H} & 3.935618 & -0.075347 & 0.695318 \\ \mathrm{H} & 2.734339 & -0.593688 & 2.003055 \\ \mathrm{O} & 0.929791 & 1.566858 & -0.142438 \\ \mathrm{C} & 1.760903 & 1.624565 & 0.787966 \\ \mathrm{H} & 2.656603 & 2.249658 & 0.667164 \\ \mathrm{H} & 1.493883 & 1.334406 & 1.810515 \\ \mathrm{H} & -3.031178 & 0.039476 & 1.397011 \\ \mathrm{C} & 2.524735 & -0.829579 & -1.456066 \\ \mathrm{H} & 2.008331 & 0.048224 & -1.867602 \\ \mathrm{H} & 3.600366 & -0.676885 & -1.575777 \\ \mathrm{H} & 2.220834 & -1.696617 & -2.050315\end{array}$

$\begin{array}{cccc}\text { 3b-p } & & & \\ \text { Pd } & 0.136459 & -0.041758 & -0.138557 \\ \text { C } & 1.748073 & -1.429914 & -0.537029 \\ \text { C } & 2.312457 & -0.199287 & -0.151757 \\ \text { C } & 1.957740 & 0.963050 & -0.860482 \\ \text { H } & 1.813855 & -2.292697 & 0.118506 \\ \text { H } & 1.556811 & -1.640915 & -1.588083 \\ \text { H } & 2.248919 & 1.934745 & -0.470071 \\ \text { H } & 1.787241 & 0.915500 & -1.935658 \\ \text { C } & -0.916872 & 1.928411 & 0.387346 \\ \text { H } & -0.883101 & 1.917611 & 1.474616 \\ \text { H } & -0.354802 & 2.713492 & -0.112269 \\ \text { C } & -1.861018 & 1.197010 & -0.287754 \\ \text { C } & -2.819394 & 0.279998 & 0.431636\end{array}$




$\begin{array}{cccc}\mathrm{H} & -3.848837 & 0.535963 & 0.143444 \\ \mathrm{H} & -2.722643 & 0.416747 & 1.513249 \\ \mathrm{O} & -1.198459 & -1.488433 & 0.434228 \\ \mathrm{C} & -2.505734 & -1.184112 & 0.096792 \\ \mathrm{H} & -2.728545 & -1.353306 & -0.976135 \\ \mathrm{H} & -3.196526 & -1.840109 & 0.655565 \\ \mathrm{H} & 2.756501 & -0.104254 & 0.837708 \\ \mathrm{C} & -2.111991 & 1.415155 & -1.756218 \\ \mathrm{H} & -3.057244 & 1.957608 & -1.885761 \\ \mathrm{H} & -1.317006 & 1.998439 & -2.227511 \\ \mathrm{H} & -2.216975 & 0.465605 & -2.290860\end{array}$

$\begin{array}{rrrr}\mathbf{4} & & & \\ \text { Pd } & -0.127589 & 0.096637 & -0.159197 \\ \text { C } & 1.472430 & -0.711356 & 1.155536 \\ \text { C } & 0.938108 & -1.728483 & 0.341016 \\ \text { C } & -0.437752 & -2.035668 & 0.332287 \\ \text { H } & 2.511073 & -0.418919 & 1.032021 \\ \text { H } & 1.036774 & -0.508535 & 2.133019 \\ \text { H } & -0.824271 & -2.730442 & -0.407341 \\ \text { H } & -1.026142 & -1.939819 & 1.243735 \\ \text { C } & -1.772906 & 0.886668 & -1.374163 \\ \text { C } & -1.284128 & 1.956239 & -0.582615 \\ \text { C } & 0.101471 & 2.244731 & -0.608452 \\ \text { H } & -2.806075 & 0.582410 & -1.235487 \\ \text { H } & -1.362867 & 0.759226 & -2.373493 \\ \text { H } & 0.540914 & 2.967190 & 0.070211 \\ \text { H } & 0.612029 & 2.137691 & -1.563109 \\ \text { H } & 1.547809 & -2.093489 & -0.485787 \\ \text { O } & -2.141159 & 2.437032 & 0.351850 \\ \text { C } & -1.614817 & 3.263044 & 1.375884 \\ \text { H } & -2.459449 & 3.516611 & 2.016857 \\ \text { H } & -0.858012 & 2.732002 & 1.964389\end{array}$




$\begin{array}{cccc}\text { H } & -1.183799 & 4.182960 & 0.965785 \\ \text { 4a-r } & & & \\ \text { Pd } & -0.032432 & -0.312672 & -0.329639 \\ \text { C } & 1.812014 & -1.687936 & -0.473018 \\ \text { C } & 2.204773 & -0.370579 & -0.228504 \\ \text { C } & 1.659243 & 0.663957 & -1.063283 \\ \text { H } & 2.044078 & -2.497239 & 0.210389 \\ \text { H } & 1.586300 & -1.975315 & -1.496432 \\ \text { H } & 1.858228 & 1.690854 & -0.766609 \\ \text { H } & 1.642932 & 0.484389 & -2.137516 \\ \text { C } & -1.235261 & 1.464246 & -0.526312 \\ \text { H } & -0.913890 & 2.121154 & 0.288494 \\ \text { H } & -0.940039 & 1.881296 & -1.492671 \\ \text { C } & -2.646266 & 1.122747 & -0.476036 \\ \text { C } & -3.473047 & 1.179212 & 0.598197 \\ \text { H } & -3.067113 & 0.708444 & -1.394985 \\ \text { H } & -4.521147 & 0.902191 & 0.516876 \\ \text { H } & -3.151003 & 1.625273 & 1.538272 \\ \text { O } & -1.696362 & -1.590461 & 0.298564 \\ \text { C } & -2.418317 & -1.292918 & 1.245363 \\ \text { H } & -3.349062 & -1.853322 & 1.422678 \\ \text { H } & -2.127850 & -0.540780 & 1.989559 \\ \text { O } & 2.843921 & 0.050723 & 0.886898 \\ \text { C } & 3.090936 & -0.904693 & 1.903511 \\ \text { H } & 3.595570 & -0.365274 & 2.705074 \\ \text { H } & 2.152903 & -1.327698 & 2.281747 \\ \text { H } & 3.739659 & -1.711207 & 1.544140\end{array}$

\section{4a-ts}

$\begin{array}{llll}\text { Pd } & -0.035151 & -0.088093 & -0.389267\end{array}$

$\begin{array}{llll}\text { C } & 1.733793 & -1.464062 & -0.502477\end{array}$

$\begin{array}{llll}\text { C } & 2.185043 & -0.152916 & -0.290097\end{array}$ 


$\begin{array}{lrrr}\mathrm{C} & 1.670570 & 0.862709 & -1.156518 \\ \mathrm{H} & 1.914755 & -2.257413 & 0.214414 \\ \mathrm{H} & 1.544517 & -1.781666 & -1.524765 \\ \mathrm{H} & 1.880027 & 1.895551 & -0.889331 \\ \mathrm{H} & 1.636837 & 0.649472 & -2.223915 \\ \mathrm{C} & -1.240029 & 1.756103 & -0.546917 \\ \mathrm{H} & -0.909467 & 2.351969 & 0.307808 \\ \mathrm{H} & -0.916828 & 2.172089 & -1.501894 \\ \mathrm{C} & -2.591095 & 1.306488 & -0.513809 \\ \mathrm{C} & -3.388402 & 1.110779 & 0.593264 \\ \mathrm{H} & -2.985377 & 0.931965 & -1.460755 \\ \mathrm{H} & -4.424690 & 0.811856 & 0.458555 \\ \mathrm{H} & -3.146474 & 1.599471 & 1.534942 \\ \mathrm{O} & -1.693587 & -1.298791 & 0.218752 \\ \mathrm{C} & -2.413050 & -0.883077 & 1.156812 \\ \mathrm{H} & -3.347600 & -1.414813 & 1.385409 \\ \mathrm{H} & -1.991095 & -0.272565 & 1.964298 \\ \mathrm{O} & 2.830601 & 0.275981 & 0.816632 \\ \mathrm{C} & 3.030757 & -0.657789 & 1.865412 \\ \mathrm{H} & 3.552681 & -0.114493 & 2.652919 \\ \mathrm{H} & 2.073450 & -1.028187 & 2.249449 \\ \mathrm{H} & 3.647727 & -1.500646 & 1.535341\end{array}$

$\begin{array}{cccc}\text { 4a-p } & & & \\ \text { Pd } & 0.069284 & -0.039731 & -0.218024 \\ \text { C } & 1.688807 & -1.389885 & -0.634883 \\ \text { C } & 2.292829 & -0.217109 & -0.122701 \\ \text { C } & 1.916906 & 0.997973 & -0.746627 \\ \text { H } & 1.713125 & -2.333189 & -0.100972 \\ \text { H } & 1.563419 & -1.462314 & -1.712931 \\ \text { H } & 2.210930 & 1.925755 & -0.262655 \\ \text { H } & 1.848253 & 1.020194 & -1.832319 \\ \text { C } & -0.941249 & 1.934193 & 0.399258\end{array}$




$\begin{array}{lrrr}\mathrm{H} & -0.855772 & 1.946661 & 1.484119 \\ \mathrm{H} & -0.409305 & 2.710972 & -0.142041 \\ \mathrm{C} & -1.890644 & 1.165549 & -0.217980 \\ \mathrm{C} & -2.845815 & 0.237308 & 0.473386 \\ \mathrm{H} & -2.051784 & 1.310269 & -1.288777 \\ \mathrm{H} & -3.877004 & 0.546661 & 0.251727 \\ \mathrm{H} & -2.697071 & 0.286490 & 1.557323 \\ \mathrm{O} & -1.258387 & -1.537462 & 0.236431 \\ \mathrm{C} & -2.579118 & -1.192198 & -0.008171 \\ \mathrm{H} & -2.847008 & -1.247154 & -1.082535 \\ \mathrm{H} & -3.252753 & -1.893492 & 0.515001 \\ \mathrm{O} & 2.927519 & -0.126366 & 1.062927 \\ \mathrm{C} & 2.942158 & -1.281215 & 1.893145 \\ \mathrm{H} & 3.478538 & -0.989216 & 2.795554 \\ \mathrm{H} & 1.923456 & -1.588326 & 2.152752 \\ \mathrm{H} & 3.469860 & -2.109500 & 1.408930\end{array}$

\section{4b-p(1)}

$\begin{array}{rrrc}\text { Pd } & -0.161085 & 0.092235 & -0.060097 \\ \mathrm{C} & -1.994694 & 1.241240 & -0.337194 \\ \mathrm{C} & -2.314318 & -0.124457 & -0.242874 \\ \mathrm{C} & -1.701404 & -1.039376 & -1.124351 \\ \mathrm{H} & -2.275304 & 1.924913 & 0.457919 \\ \mathrm{H} & -1.781207 & 1.692745 & -1.304759 \\ \mathrm{H} & -1.832604 & -2.105124 & -0.952150 \\ \mathrm{H} & -1.512038 & -0.747477 & -2.157432 \\ \mathrm{C} & 1.346556 & -1.544502 & 0.340036 \\ \mathrm{H} & 1.391930 & -1.384958 & 1.413558 \\ \mathrm{H} & 0.958996 & -2.500855 & 0.007348 \\ \mathrm{C} & 2.136896 & -0.758632 & -0.467414 \\ \mathrm{C} & 2.921465 & 0.429926 & 0.016932 \\ \mathrm{H} & 3.036929 & 1.115511 & -0.827871 \\ \mathrm{H} & 3.925942 & 0.080041 & 0.296721\end{array}$




$\begin{array}{lccc}\mathrm{O} & 0.957254 & 1.581756 & 0.799777 \\ \mathrm{C} & 2.222860 & 1.160056 & 1.163283 \\ \mathrm{H} & 2.841257 & 2.032388 & 1.435882 \\ \mathrm{H} & 2.223709 & 0.506265 & 2.060299 \\ \mathrm{H} & -2.784580 & -0.496698 & 0.665980 \\ \mathrm{O} & 2.397950 & -1.021439 & -1.762175 \\ \mathrm{C} & 1.837694 & -2.189720 & -2.339616 \\ \mathrm{H} & 2.162592 & -2.194463 & -3.379811 \\ \mathrm{H} & 2.203735 & -3.091890 & -1.837521 \\ \mathrm{H} & 0.743714 & -2.159253 & -2.295209\end{array}$

\section{4b-p(2)}

$\begin{array}{rrrc}\text { Pd } & 0.099469 & 0.074361 & 0.001681 \\ \mathrm{C} & -1.603463 & 1.234096 & -0.751599 \\ \mathrm{C} & -1.992075 & -0.106090 & -0.584923 \\ \mathrm{C} & -1.251062 & -1.127210 & -1.214659 \\ \mathrm{H} & -2.016296 & 2.007527 & -0.111411 \\ \mathrm{H} & -1.179341 & 1.570576 & -1.696526 \\ \mathrm{H} & -1.448606 & -2.164103 & -0.955091 \\ \mathrm{H} & -0.829079 & -0.963667 & -2.205835 \\ \mathrm{C} & 1.364777 & -1.582420 & 0.828760 \\ \mathrm{H} & 1.269970 & -1.324059 & 1.878926 \\ \mathrm{H} & 0.990738 & -2.548734 & 0.504995 \\ \mathrm{C} & 2.294610 & -0.938533 & 0.045009 \\ \mathrm{C} & 3.103448 & 0.249558 & 0.503713 \\ \mathrm{H} & 3.353697 & 0.882327 & -0.351425 \\ \mathrm{H} & 4.049662 & -0.124281 & 0.924676 \\ \mathrm{O} & 1.156875 & 1.590320 & 0.901914 \\ \mathrm{C} & 2.319574 & 1.122819 & 1.485463 \\ \mathrm{H} & 2.963616 & 1.972053 & 1.771757 \\ \mathrm{H} & 2.144402 & 0.553737 & 2.421154 \\ \mathrm{H} & -2.649779 & -0.365427 & 0.243394 \\ \mathrm{O} & 2.614842 & -1.558890 & -1.117975\end{array}$




$\begin{array}{llll}\mathrm{C} & 3.225092 & -0.809741 & -2.160970 \\ \mathrm{H} & 3.255512 & -1.480953 & -3.019752 \\ \mathrm{H} & 2.631935 & 0.075426 & -2.414188 \\ \mathrm{H} & 4.246181 & -0.511554 & -1.904861\end{array}$

$\begin{array}{cccc}5 & & & \\ \text { Pt } & 0.000000 & 0.000000 & 0.000000 \\ \text { C } & 1.595473 & -1.094101 & 0.995249 \\ \text { C } & 0.898786 & -1.935619 & 0.088311 \\ \text { C } & -0.507641 & -2.107578 & 0.181754 \\ \text { H } & 2.637296 & -0.865017 & 0.790297 \\ \text { H } & 1.310718 & -1.069095 & 2.045871 \\ \text { H } & -1.018813 & -2.626018 & -0.624122 \\ \text { H } & -0.985514 & -2.176480 & 1.157748 \\ \text { C } & -1.595473 & 1.094101 & -0.995249 \\ \text { C } & -0.898786 & 1.935619 & -0.088311 \\ \text { C } & 0.507641 & 2.107578 & -0.181754 \\ \text { H } & -2.637296 & 0.865017 & -0.790297 \\ \text { H } & -1.310718 & 1.069095 & -2.045871 \\ \text { H } & 1.018813 & 2.626018 & 0.624122 \\ \mathrm{H} & 0.985514 & 2.176480 & -1.157748 \\ \text { H } & 1.390708 & -2.207273 & -0.845014 \\ \mathrm{H} & -1.390708 & 2.207273 & 0.845014 \\ \mathrm{XX} & 0.970701 & -0.237149 & -0.038743\end{array}$

$\begin{array}{cccc}\mathbf{5 - r} & & & \\ \text { Pt } & -0.507788 & -0.020246 & -0.038124 \\ \text { C } & -2.451988 & 1.062858 & -0.231058 \\ \text { C } & -2.528766 & -0.083951 & 0.576861 \\ \text { C } & -2.085165 & -1.348675 & 0.039037 \\ \text { H } & -2.591936 & 2.039810 & 0.223971 \\ \text { H } & -2.629486 & 1.005461 & -1.302878 \\ \text { H } & -2.022474 & -2.190825 & 0.725287\end{array}$




$\begin{array}{lrrc}\mathrm{H} & -2.375545 & -1.621660 & -0.976238 \\ \mathrm{H} & -2.664574 & 0.026524 & 1.651341 \\ \mathrm{C} & 1.036623 & -1.497841 & -0.007406 \\ \mathrm{H} & 1.078056 & -1.865617 & 1.024631 \\ \mathrm{H} & 0.672800 & -2.297387 & -0.661055 \\ \mathrm{C} & 2.332483 & -0.997975 & -0.462413 \\ \mathrm{C} & 3.356782 & -0.551430 & 0.303498 \\ \mathrm{H} & 2.447895 & -0.892227 & -1.543369 \\ \mathrm{H} & 4.281473 & -0.196514 & -0.144810 \\ \mathrm{H} & 3.346359 & -0.668125 & 1.386276 \\ \mathrm{O} & 0.938836 & 1.515787 & -0.339884 \\ \mathrm{C} & 1.905472 & 1.670322 & 0.414024 \\ \mathrm{H} & 2.712628 & 2.350069 & 0.112387 \\ \mathrm{H} & 1.918138 & 1.263798 & 1.430277\end{array}$

$\begin{array}{cccc}\text { 5-ts } & & & \\ \text { Pt } & -0.355137 & -0.002060 & -0.048222 \\ \text { C } & -2.255365 & 1.088580 & -0.183142 \\ \text { C } & -2.358349 & -0.089523 & 0.587971 \\ \text { C } & -1.950162 & -1.333592 & -0.005798 \\ \text { H } & -2.362397 & 2.050969 & 0.310265 \\ \text { H } & -2.472719 & 1.076095 & -1.249427 \\ \text { H } & -1.887711 & -2.205324 & 0.642268 \\ \text { H } & -2.242682 & -1.555790 & -1.032446 \\ \text { H } & -2.481650 & -0.018059 & 1.667113 \\ \text { C } & 1.176615 & -1.546151 & -0.004477 \\ \text { H } & 1.184292 & -1.880648 & 1.037882 \\ \text { H } & 0.792500 & -2.324879 & -0.667180 \\ \text { C } & 2.425722 & -0.989934 & -0.449350 \\ \text { C } & 3.366871 & -0.349701 & 0.314418 \\ \text { H } & 2.558114 & -0.926967 & -1.531353 \\ \text { H } & 4.286674 & 0.005660 & -0.142883 \\ \text { H } & 3.380335 & -0.479200 & 1.394685\end{array}$




$\begin{array}{llll}\mathrm{O} & 1.100222 & 1.473368 & -0.368571 \\ \mathrm{C} & 2.100440 & 1.578468 & 0.384061 \\ \mathrm{H} & 2.910392 & 2.248325 & 0.072795 \\ \mathrm{H} & 2.033440 & 1.329481 & 1.447495\end{array}$

$\begin{array}{cccc}\text { 5-p } & & & \\ \text { Pt } & -0.309886 & -0.041929 & 0.027885 \\ \text { C } & -1.984722 & -1.366864 & 0.024129 \\ \text { C } & -2.414690 & -0.074506 & -0.386389 \\ \text { C } & -2.146582 & 1.005621 & 0.493791 \\ \text { H } & -1.968705 & -2.177936 & -0.698118 \\ \text { H } & -2.101753 & -1.665610 & 1.064910 \\ \text { H } & -2.310923 & 2.017505 & 0.131167 \\ \text { H } & -2.267247 & 0.873347 & 1.568186 \\ \text { H } & -2.665167 & 0.118410 & -1.427278 \\ \text { C } & 0.663403 & 1.861125 & -0.470616 \\ \text { H } & 0.812784 & 1.870548 & -1.548643 \\ \text { H } & 0.087263 & 2.687102 & -0.063363 \\ \text { C } & 1.521899 & 1.130075 & 0.348063 \\ \text { C } & 2.643582 & 0.261450 & -0.158876 \\ \text { H } & 1.536545 & 1.365593 & 1.413444 \\ \text { H } & 3.602990 & 0.618034 & 0.238246 \\ \text { H } & 2.686402 & 0.309188 & -1.252269 \\ \text { O } & 1.061423 & -1.523681 & -0.127627 \\ \text { C } & 2.354999 & -1.173463 & 0.272396 \\ \text { H } & 2.482381 & -1.249275 & 1.367820 \\ \text { H } & 3.073603 & -1.871549 & -0.183306\end{array}$

$\begin{array}{crrr}\text { sz-1 } & & & \\ \text { Pd } & -0.490934 & -0.007231 & 0.085143 \\ \text { C } & -2.519111 & 0.967469 & -0.396297 \\ \text { C } & -2.670022 & -0.208184 & 0.343830 \\ \text { C } & -2.119806 & -1.440910 & -0.085757\end{array}$




$\begin{array}{lccc}\mathrm{H} & -2.834005 & 1.916882 & 0.027205 \\ \mathrm{H} & -2.426155 & 0.931375 & -1.480628 \\ \mathrm{H} & -2.157577 & -2.297915 & 0.580470 \\ \mathrm{H} & -2.080432 & -1.675020 & -1.149174 \\ \mathrm{H} & -2.981935 & -0.121641 & 1.384478 \\ \mathrm{C} & 1.032531 & -1.478437 & 0.389236 \\ \mathrm{H} & 1.327328 & -1.404806 & 1.442155 \\ \mathrm{H} & 0.546730 & -2.441442 & 0.211529 \\ \mathrm{C} & 2.159758 & -1.262897 & -0.528423 \\ \mathrm{C} & 3.378544 & -0.776922 & -0.217689 \\ \mathrm{H} & 1.974834 & -1.510425 & -1.576351 \\ \mathrm{H} & 4.144027 & -0.636551 & -0.976564 \\ \mathrm{H} & 3.653195 & -0.543011 & 0.810101 \\ \mathrm{P} & 1.040948 & 1.754364 & 0.056942 \\ \mathrm{H} & 2.195672 & 1.661593 & 0.861128 \\ \mathrm{H} & 0.688002 & 3.090941 & 0.360275 \\ \mathrm{H} & 1.691793 & 2.009922 & -1.168127\end{array}$

sz-2

$\begin{array}{rrrr}\mathrm{C} & -2.446508 & 0.880343 & -0.313564 \\ \mathrm{C} & -2.475019 & -0.290479 & 0.450754 \\ \mathrm{C} & -1.889063 & -1.497933 & -0.000865 \\ \mathrm{Pd} & -0.336232 & 0.034983 & 0.043225 \\ \mathrm{P} & 1.064180 & 1.880251 & -0.215208 \\ \mathrm{C} & 1.258288 & -1.348481 & 0.334699 \\ \mathrm{C} & 1.732541 & -1.120958 & 1.707491 \\ \mathrm{C} & 1.436943 & -1.857041 & 2.795085 \\ \mathrm{H} & -2.791163 & 1.815062 & 0.119742 \\ \mathrm{H} & -2.435029 & 0.829957 & -1.401213 \\ \mathrm{H} & -1.825453 & -2.341300 & 0.680364 \\ \mathrm{H} & -1.907086 & -1.748223 & -1.060935 \\ \mathrm{H} & -2.713754 & -0.203760 & 1.510564 \\ \mathrm{H} & 2.379459 & -0.252131 & 1.858088\end{array}$




$\begin{array}{rrrr}\mathrm{H} & 1.819512 & -1.597803 & 3.778516 \\ \mathrm{H} & 0.802860 & -2.739507 & 2.726682 \\ \mathrm{H} & 2.465166 & 1.719552 & -0.323967 \\ \mathrm{H} & 1.085544 & 2.887361 & 0.776282 \\ \mathrm{H} & 0.903469 & 2.740817 & -1.324614 \\ \mathrm{H} & 2.020637 & -1.123003 & -0.418738 \\ \mathrm{H} & 0.885111 & -2.363795 & 0.184355\end{array}$

\begin{tabular}{|c|c|c|c|}
\hline \multicolumn{4}{|c|}{ SZ-r } \\
\hline $\mathrm{C}$ & -2.463516 & 0.753172 & -0.341188 \\
\hline $\mathrm{Pd}$ & -0.373691 & -0.098332 & 0.055225 \\
\hline $\mathrm{C}$ & 1.258706 & -1.469630 & 0.209864 \\
\hline $\mathrm{C}$ & 1.822234 & -1.387589 & 1.560389 \\
\hline $\mathrm{C}$ & 1.625842 & -2.250459 & 2.579376 \\
\hline $\mathrm{C}$ & -2.522029 & -0.486319 & 0.301442 \\
\hline $\mathrm{C}$ & -1.889045 & -1.633666 & -0.238057 \\
\hline $\mathrm{P}$ & 0.905116 & 1.847806 & 0.210961 \\
\hline $\mathrm{H}$ & -2.830699 & 1.639103 & 0.168892 \\
\hline $\mathrm{H}$ & -2.390026 & 0.813472 & -1.426003 \\
\hline $\mathrm{H}$ & -1.847749 & -2.543432 & 0.354045 \\
\hline $\mathrm{H}$ & -1.847039 & -1.774491 & -1.317659 \\
\hline $\mathrm{H}$ & -2.808006 & -0.498419 & 1.352355 \\
\hline $\mathrm{H}$ & 2.464289 & -0.525292 & 1.762337 \\
\hline $\mathrm{H}$ & 2.108665 & -2.110819 & 3.543446 \\
\hline $\mathrm{H}$ & 1.016504 & -3.144340 & 2.455124 \\
\hline $\mathrm{H}$ & 2.319060 & 1.825926 & 0.171188 \\
\hline $\mathrm{H}$ & 0.768703 & 2.610330 & 1.390915 \\
\hline $\mathrm{H}$ & 0.729301 & 2.904728 & -0.710510 \\
\hline $\mathrm{H}$ & 1.972005 & -1.141445 & -0.554468 \\
\hline $\mathrm{H}$ & 0.899771 & -2.473225 & -0.029466 \\
\hline $\mathrm{C}$ & -0.370813 & -0.216283 & 3.857762 \\
\hline $\mathrm{O}$ & -0.905820 & 0.678079 & 3.240485 \\
\hline $\mathrm{H}$ & 0.566558 & -0.055307 & 4.423718 \\
\hline
\end{tabular}


$\begin{array}{llll}\mathrm{H} & -0.797670 & -1.235660 & 3.904085\end{array}$

sz-ts

$\begin{array}{rrrc}\mathrm{C} & -2.374399 & 0.836399 & -0.696909 \\ \mathrm{Pd} & -0.622125 & -0.248703 & 0.166770 \\ \mathrm{C} & 0.817583 & -1.888605 & 0.450544 \\ \mathrm{C} & 1.746749 & -1.512698 & 1.450632 \\ \mathrm{C} & 1.632583 & -1.766048 & 2.812529 \\ \mathrm{C} & -2.754035 & -0.449948 & -0.279127 \\ \mathrm{C} & -2.084995 & -1.589915 & -0.768080 \\ \mathrm{P} & 0.496150 & 1.643666 & 0.985063 \\ \mathrm{H} & -2.793749 & 1.705403 & -0.197689 \\ \mathrm{H} & -1.998673 & 0.999448 & -1.705933 \\ \mathrm{H} & -2.291448 & -2.560250 & -0.325774 \\ \mathrm{H} & -1.702973 & -1.605000 & -1.788095 \\ \mathrm{H} & -3.359729 & -0.546612 & 0.620613 \\ \mathrm{H} & 2.594321 & -0.904010 & 1.126272 \\ \mathrm{H} & 2.530338 & -1.700570 & 3.422934 \\ \mathrm{H} & 0.908802 & -2.518176 & 3.122415 \\ \mathrm{H} & 1.672843 & 1.621568 & 1.758263 \\ \mathrm{H} & -0.266541 & 2.589118 & 1.688824 \\ \mathrm{H} & 0.993378 & 2.531077 & -0.014741 \\ \mathrm{H} & 1.210407 & -1.906976 & -0.569486 \\ \mathrm{H} & 0.196362 & -2.751054 & 0.691728 \\ \mathrm{C} & 0.767916 & -0.096005 & 3.665748 \\ \mathrm{O} & -0.201104 & 0.392882 & 3.029346 \\ \mathrm{H} & 1.724201 & 0.460887 & 3.717685 \\ & 0.568790 & -0.707970 & 4.560726\end{array}$

$\begin{array}{cccc}\text { sz-p } & & & \\ \text { C } & -2.506930 & 0.631802 & -0.342462 \\ \text { Pd } & -0.413662 & 0.090136 & -0.068087 \\ \text { C } & -2.438309 & -0.592736 & 0.361552\end{array}$




$\begin{array}{lrrr}\mathrm{C} & -1.741628 & -1.668978 & -0.204752 \\ \mathrm{P} & 0.583220 & 2.191396 & 0.213828 \\ \mathrm{H} & -2.923893 & 1.503977 & 0.155259 \\ \mathrm{H} & -2.592188 & 0.626311 & -1.428408 \\ \mathrm{H} & -1.476248 & -2.531210 & 0.398771 \\ \mathrm{H} & -1.729349 & -1.809135 & -1.283204 \\ \mathrm{H} & -2.659231 & -0.605855 & 1.427549 \\ \mathrm{H} & 1.818338 & 2.216604 & 0.893843 \\ \mathrm{H} & -0.071321 & 3.195263 & 0.962324 \\ \mathrm{H} & 0.946848 & 3.009954 & -0.879475 \\ \mathrm{O} & 1.325891 & -0.966547 & 0.284750 \\ \mathrm{C} & 2.447221 & -0.722066 & -0.472921 \\ \mathrm{H} & 2.800185 & 0.335141 & -0.420499 \\ \mathrm{H} & 3.278411 & -1.327993 & -0.069240 \\ \mathrm{C} & 2.311395 & -1.082856 & -1.968773 \\ \mathrm{H} & 1.938251 & -2.109812 & -2.044747 \\ \mathrm{H} & 3.314359 & -1.055311 & -2.419544 \\ \mathrm{C} & 1.423265 & -0.145899 & -2.717206 \\ \mathrm{C} & 0.258793 & -0.454961 & -3.299428 \\ \mathrm{H} & 1.767814 & 0.889982 & -2.774595 \\ \mathrm{H} & -0.332869 & 0.290508 & -3.825905 \\ \mathrm{H} & -0.121131 & -1.475059 & -3.292317\end{array}$

